\title{
Prospective population studies of incident heart failure without data on baseline left ventricular ejection fraction
}

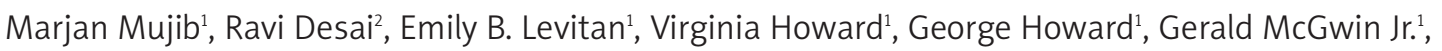
Ali Ahmed ${ }^{1,3}$

1University of Alabama at Birmingham, Birmingham, AL, USA
2Lehigh Valley Hospital, Allentown, Pennsylvania, USA

3Veterans Affairs Medical Center, Birmingham, AL, USA

Submitted: 25 September 2010

Accepted: 11 October 2010

Arch Med Sci 2010; 6, 5: 686-688

DOI: $10.5114 /$ aoms.2010.17081

Copyright (c) 2010 Termedia \& Banach

\author{
Corresponding author: \\ Ali Ahmed MD, MPH \\ University of Alabama at \\ Birmingham \\ $15303^{\text {rd }}$ Ave South, $\mathrm{CH}-19$, \\ Ste-219 \\ Birmingham AL 35294-2041 \\ Phone: +1 2059349632 \\ Fax: +1 2059757099 \\ E-mail: aahmed@uab.edu
}

\begin{abstract}
Introduction: Left ventricular ejection fraction (LVEF) is a predictor of incident heart failure (HF). However, baseline LVEF is often unavailable in population studies of HF.

Material and methods: Of the 5324 Cardiovascular Health Study (CHS) participants free of baseline HF, 143 (3\%) had LVEF < 45\% and 1091 (21\%) developed HF during 13 years of follow-up. Using public-use copies of the CHS data, we compared two predictor models of incident HF, with and without adjustment for baseline LVEF.

Results: Baseline impaired LVEF was a strong independent predictor of incident HF (adjusted hazard ratio, 2.78; $P<0.001$ ) but had no impact on the direction, magnitude or significance of independent associations of the other predictors of incident HF such as age, sex, race, coronary artery disease, hypertension and diabetes.

Conclusion: Baseline LVEF is an important predictor for incident HF but is not essential in population studies of risk factors for incident HF.
\end{abstract}

Key words: heart failure, left ventricular ejection fraction, epidemiology, population studies.

\section{Introduction}

Left ventricular (LV) systolic dysfunction (SD) is an important predictor of incident heart failure (HF) [1]. Therefore, it may be important to collect and adjust for data on baseline LV ejection fraction (LVEF) in studies of incident HF. However, cost and other logistical considerations often preclude collection of baseline data on LVEF in large population studies of incident HF [2]. The methodological impact of the lack of baseline LVEF data on the interpretation of the findings from studies of incident HF is unknown. We used public-use copies of the Cardiovascular Health Study (CHS) datasets obtained from the National Heart, Lung, and Blood Institute to compare two multivariable Cox regression models of incident HF with and without adjustment for baseline LVEF.

\section{Material and methods}

The CHS is an ongoing epidemiologic study of cardiovascular disease in community-dwelling older adults in the United States; the details of the 
rationale, design and implementation of which have been previously reported $[1,3,4]$. Briefly, an original cohort of 5201 participants recruited during 19891990, was complemented by a second cohort of 687 African-American participants recruited during 1992-1993. Of 5888 CHS participants, data on 5795 participants were available in the public-use copy of the dataset (93 participants did not consent to be included in the de-identified public-use data). Of the 5324 CHS participants who were free of baseline HF (centrally adjudicated) and also had echocardiographic data on baseline LVEF, 143 (2\%) had LVSD, defined as LVEF $<45 \%$.

Incident HF was centrally adjudicated during a median follow-up of 12 years. The process of adjudication of HF in CHS has been welldocumented in the literature [5-7]. Briefly, participants were asked about physician-diagnosed HF during semi-annual visits. The CHS Events Committee later adjudicated the diagnosis of $\mathrm{HF}$ through the examination of participants' medical records for a constellation of symptoms, physical signs, and other supporting findings suggestive of $\mathrm{HF}$, use of medications commonly used for $\mathrm{HF}$, and follow-up surveillance. Two multivariable Cox regression models were developed to identify predictors of incident HF. In both models, incident HF was the dependent variable, and various demographic, clinical and laboratory variables were entered as covariates. The models were similar except that in one model an additional variable for LVSD was entered as a covariate.

\section{Resutlts}

Participants $(n=5324)$ had a mean age of $73( \pm 6)$ years, 58\% were women and $13 \%$ were African American. Overall, 1091 (21\%) participants developed incident HF during 50,143 person-years of follow-up. Baseline LVSD had an independent association with incident HF (adjusted hazard ratio, 2.78; $P<0.001$; Table I). Adjustment for LVSD, however, had no impact on the direction, magnitude or significance of the independent associations of other predictors with incident HF. For example, adjusted hazard ratios for age $\geq 75$ years, before and after LVSD adjustments, were 1.95 and 1.94 respectively (both $P<0.001$; Table I). Adjusted hazard ratios for other predictors of incident $\mathrm{HF}$ were also similar regardless of adjustment for baseline LVSD (Table I).

Table I. Adjusted hazard ratios (HR) for predictors of incident heart failure (HF) in community-dwelling older adults without prevalent HF, with and without adjustment for baseline left ventricular systolic dysfunction (LVSD)

\begin{tabular}{|c|c|c|c|c|c|}
\hline \multirow[t]{2}{*}{ Variable } & \multicolumn{2}{|c|}{ Model without LVSD } & \multicolumn{2}{|c|}{ Model with LVSD } & \multirow{2}{*}{$\begin{array}{c}\text { Absolute } \\
\text { difference in HR }\end{array}$} \\
\hline & Adjusted HR & $P$ value & Adjusted HR & $P$ value & \\
\hline Age $\geq 75$ years & 1.95 & $<0.001$ & 1.94 & $<0.001$ & 0.01 \\
\hline Female gender & 0.74 & $<0.001$ & 0.76 & $<0.001$ & 0.02 \\
\hline African American & 0.66 & $<0.001$ & 0.67 & $<0.001$ & 0.01 \\
\hline Current smoking & 1.39 & $<0.001$ & 1.42 & $<0.001$ & 0.03 \\
\hline General health fair to poor & 1.41 & $<0.001$ & 1.40 & $<0.001$ & 0.01 \\
\hline Coronary artery disease & 1.75 & $<0.001$ & 1.66 & $<0.001$ & 0.09 \\
\hline Hypertension & 1.24 & 0.010 & 1.27 & 0.004 & 0.03 \\
\hline Diabetes mellitus & 1.75 & $<0.001$ & 1.78 & $<0.001$ & 0.03 \\
\hline Stroke & 1.30 & 0.056 & 1.34 & 0.033 & 0.04 \\
\hline Chronic obstructive pulmonary disease & 1.23 & 0.017 & 1.23 & 0.017 & 0.00 \\
\hline Atrial fibrillation & 2.44 & $<0.001$ & 2.31 & $<0.001$ & 0.13 \\
\hline Left ventricular hypertrophy & 1.77 & $<0.001$ & 1.72 & $<0.001$ & 0.05 \\
\hline Systolic blood pressure [mmHg] & 1.01 & $<0.001$ & 1.01 & $<0.001$ & 0.00 \\
\hline Peripheral arterial disease & 1.61 & $<0.001$ & 1.59 & $<0.001$ & 0.02 \\
\hline Serum creatinine $[\mathrm{mg} / \mathrm{dl}]$ & 1.39 & $<0.001$ & 1.40 & $<0.001$ & 0.01 \\
\hline Serum uric acid [mg/dl] & 1.09 & $<0.001$ & 1.09 & $<0.001$ & 0.00 \\
\hline Serum albumin [g/dl] & 0.71 & 0.002 & 0.69 & 0.002 & 0.02 \\
\hline Serum insulin $[\mu \mathrm{U} / \mathrm{ml}]$ & 1.003 & 0.016 & 1.002 & 0.024 & 0.001 \\
\hline C-reactive protein $[\mathrm{mg} / \mathrm{dl}]$ & 1.01 & $<0.001$ & 1.01 & $<0.001$ & 0.00 \\
\hline Hemoglobin [g/dl] & 0.94 & 0.013 & 0.94 & 0.016 & 0.00 \\
\hline LVSD (ejection fraction < 45\%) & --- & --- & 2.78 & $<0.001$ & --- \\
\hline
\end{tabular}




\section{Discussion}

Findings from this prospective population study of incident $\mathrm{HF}$ demonstrate that the prevalence of LVSD was low among community-dwelling older adults without prevalent HF. Although baseline LVSD was a strong independent predictor of incident $\mathrm{HF}$, the predictive significance of age, female sex, African American race, coronary artery disease, diabetes and others remained unchanged before and after adjusting for LVSD. Atrial fibrillation was the only significant predictor of incident HF that had a $>5 \%$ change with additional adjustment of LVSD; however, it was strongly significant both before and after adjustment of LVSD.

Findings of the current analysis suggest that LVSD had no noticeable impact on the direction, magnitude or significance of the independent associations of other predictors of incident HF. Because LVSD is a very strong predictor of incident $\mathrm{HF}$, the absence baseline LVSD data may be viewed as a limitation in population studies of incident HF. However, findings from our study suggest that in addition to re-establishing the fact that LVSD is a strong predictor of incident HF, the costly collection of baseline LVEF data is unlikely to add any additional value in studies of other risk factors of HF. In conclusion, the methodological impact of the lack of baseline LVEF data on the interpretation of the findings from studies of incident $\mathrm{HF}$ is negligible, and the lack of baseline LVSD data is not a major limitation for population studies of predictors of incident HF.

\section{Acknowledgement}

Dr. Ahmed is supported by the National Institutes of Health through grants (R01-HL085561 and R01-HL097047) from the National Heart, Lung, and Blood Institute (NHLBI) and a generous gift from Ms. Jean B. Morris of Birmingham, Alabama.

The Cardiovascular Health Study (CHS) was conducted and supported by the NHLBI in collaboration with the CHS Investigators. This manuscript was prepared using a limited access dataset obtained by the NHLBI and does not necessarily reflect the opinions or views of the CHS Study or the NHLBI.

\section{References}

1. Gottdiener JS, Arnold AM, Aurigemma GP, et al. Predictors of congestive heart failure in the elderly: the Cardiovascular Health Study. J Am Coll Cardiol 2000; 35: 1628-37.

2. Vasan RS, Sullivan LM, D'Agostino RB, et al. Serum insulinlike growth factor I and risk for heart failure in elderly individuals without a previous myocardial infarction: the Framingham Heart Study. Ann Intern Med 2003; 139: 642-8.
3. Fried LP, Borhani NO, Enright P, et al. The Cardiovascular Health Study: design and rationale. Ann Epidemiol 1991; 1: 263-76.

4. Gardin JM, Arnold A, Gottdiener JS, et al. Left ventricular mass in the elderly. The Cardiovascular Health Study. Hypertension 1997; 29: 1095-103.

5. Psaty BM, Kuller LH, Bild D, et al. Methods of assessing prevalent cardiovascular disease in the Cardiovascular Health Study. Ann Epidemiol 1995; 5: 270-7.

6. Schellenbaum GD, Heckbert SR, Smith NL, et al. Congestive heart failure incidence and prognosis: case identification using central adjudication versus hospital discharge diagnoses. Ann Epidemiol 2006; 16: 115-22.

7. Schellenbaum GD, Rea TD, Heckbert SR, et al. Survival associated with two sets of diagnostic criteria for congestive heart failure. Am J Epidemiol 2004; 160: 628-35. 